\title{
Efeitos do Índice Glicêmico no Balanço Energético
}

revisão

\section{ANA P.M. GuttierRes Rita de Cássia G. Alfenas}

Departamento de Nutrição e Saúde, Universidade Federal de Viçosa, MG.

\section{RESUMO}

A prevalência da obesidade vem crescendo nas últimas décadas. Associado a este fato, tem-se observado uma mudança no padrão dietético da população em geral, no que diz respeito a um maior consumo de carboidratos. Segundo alguns autores, o índice glicêmico (IG) dos alimentos afeta a composição e o peso corporal. A presente revisão de literatura teve como objetivo avaliar os efeitos do IG sobre apetite, saciedade e composição corporal. A partir das evidências científicas analisadas, foi possível constatar que a maioria dos estudos que atribuem efeitos positivos ao IG é cercada de limitações metodológicas. Estudos bem delineados não observaram benefícios do IG sobre os parâmetros citados acima. Diante disso, conclui-se que o IG apresenta pouca aplicabilidade na prática clínica, como uma ferramenta capaz de controlar a saciedade, reduzir o apetite e, conseqüentemente, a prevalência de obesidade. (Arq Bras Endocrinol Metab 2007;51/3:382-388)

Descritores: Índice glicêmico; Saciedade; Apetite; Composição corporal

\section{ABSTRACT}

\section{Effects of Glycemic Index on Energy Balance.}

The prevalence of obesity has increased over the last decades. Associated to this, there has been observed a chance in the dietetic pattern of the population in general, related to the increase in carbohydrate consumption. According to some authors, the glycemic index (GI) of food may affect body composition and body weight. The purpose of this review was to evaluate the effects of Gl on appetite, satiety, and body composition. Based on the scientific evidences reviewed, it was possible to verify that the majority of the studies that observed a positive effect of Gl in that matter have a lot of methodological limitations. Well-designed studies have not observed any benefit of GI on these parameters. Therefore, it is concluded that Gl has little application in clinical practice, as a useful tool to control satiety, reduce appetite, and consequently, to reduce the prevalence of obesity. (Arq Bras Endocrinol Metab 2007;51/3:382-388)

Keywords: Glycemic index; Satiety; Appetite; Body composition

Eegundo alguns autores, as alterações na prática dietética, associadas $\checkmark$ à diminuição da atividade física, são os maiores responsáveis ambientais para o atual aumento da prevalência da obesidade (1). Há alguns anos, acreditava-se que a alta ingestão de gorduras era um dos fatores que mais contribuíam para tal. No entanto, hoje sabe-se que a redução da quantidade de gordura ingerida não resulta necessariamente na diminuição da prevalência da obesidade, já que tal medida está, na maioria das vezes, associada ao aumento do consumo de carboidratos. Segundo alguns autores, o
Recebido em 23/05/06 Revisado em 11/12/06 Aceito em 17/12/06 
aumento do consumo de carboidratos complexos contribui significativamente para o aumento da obesidade (2). Já para outros autores, o aumento da obesidade se associa ao aumento concomitante do consumo de alimentos de alto índice glicêmico (IG) na dieta americana $(1,3)$.

O IG é um parâmetro utilizado para classificar os alimentos contendo carboidratos de acordo com a resposta glicêmica que os mesmos promovem, em relação à resposta observada após consumo de um alimento de referência (pão branco ou glicose). É definido como a área formada abaixo da curva de resposta glicêmica após o consumo de $50 \mathrm{~g}$ de carboidratos de um alimento-teste, dividida pela área abaixo da curva de resposta glicêmica após o consumo do alimento de referência contendo o mesmo teor de carboidratos (4). Estudos clínicos $(3,5,7,8)$ sugerem que o IG apresenta um papel importante na regulação do peso corporal. Porém, tais estudos em geral apresentam falhas metodológicas, as quais não permitem que se chegue a uma conclusão definitiva a respeito do real papel do IG neste sentido (5).

Alguns autores (6) constataram que as dietas de alto IG apresentam menor poder de saciedade, resultando em excessiva ingestão alimentar, favorecendo o aumento do peso corporal. Além disso, o consumo de tais dietas pode alterar o perfil lipídico e a secreção insulínica, favorecendo o aparecimento de doenças cardiovasculares e de diabetes mellitus. Tem sido sugerido (7) que tal ingestão aumenta a secreção de insulina, a qual é considerada como um fator de risco independente para o ganho de peso. O consumo de alimentos de alto IG parece desencadear uma seqüência de eventos hormonais, que limita a disponibilidade de combustível metabólico no período pós-prandial, levando à fome e à ingestão alimentar excessiva (3).

Por outro lado, a ingestão de alimentos de baixo IG pode diminuir a secreção de hormônios contra-regulatórios proteolíticos como o cortisol, hormônio do crescimento e glucagon, estimulando a síntese protéica (3). Segundo alguns autores (8), a regulação da massa de gordura corporal associada à ingestão de dietas de baixo IG, pode estar relacionada à ativação de genes como o $o b$. Argumenta-se que a ingestão de tais dietas parece diminuir a expressão desses genes, diminuindo a secreção insulínica pósprandial. Por esse motivo, observou-se que a ingestão de alimentos de baixo IG tende a aumentar o teor de massa magra e a diminuir, significativamente, o teor de massa gordurosa corporal (8).

Apesar dos resultados promissores desse último estudo, deve-se ter em mente que o IG dos alimentos pode ser influenciado por uma série de fatores. Os fatores que afetam a motilidade intestinal e a secreção de insulina apresentam um efeito direto sobre este parâmetro. A proporção entre os tipos de carboidratos (amilose ou amilopectina) ingeridos, o teor de fibras e de macronutrientes que compõem os alimentos da refeição (4), o grau de processamento do grânulo de amido, o método e o tempo de cocção são alguns dos fatores passíveis de exercer influências sobre o IG (1). Assim, a interação entre todos estes fatores pode afetar drasticamente os valores do IG previstos para os alimentos ingeridos em determinadas refeições.

Geralmente, os estudos (3) que revelam menor percepção de fome e menor ingestão voluntária após a ingestão de alimento de baixo IG, em relação aos de alto IG, tiveram curta duração (um dia). Alguns estudos têm demonstrado que a obtenção de menor ingestão calórica em uma refeição subseqüente pode resultar em compensação calórica quando a ingestão é avaliada pelo restante do dia (9). Além disso, nesses estudos $(3,7,8,12)$ outros fatores como a composição de macronutrientes, a quantidade de fibras e/ou a densidade energética dos alimentos testados também diferiu. Para se estudar o efeito do IG por si, todos esses fatores dietéticos devem ser mantidos constantes (10). Estudos em que tais fatores foram controlados, sendo conduzidos em condições experimentais reproduzindo ao máximo a situação de vida livre, em que os alimentos diferindo em IG foram ingeridos ad libitum, não suportam os resultados benéficos da ingestão de alimentos de baixo IG sobre o apetite, a saciedade e a composição corporal $(10,11)$.

Diante de tais controvérsias, a presente revisão de literatura objetivou analisar as evidências científicas apresentadas em artigos publicados, buscando elucidar os reais efeitos que o IG dos alimentos exerce sobre o apetite, a saciedade e a composição corporal.

\section{REVISĀO DE LITERATURA}

Vários estudos têm sido realizados com o objetivo de identificar o papel do IG dos alimentos sobre o apetite, a saciedade $\mathrm{e}$ a manifestação da obesidade. $\mathrm{O}$ resumo de alguns desses estudos que avaliaram o efeito do IG dos alimentos na composição corporal e em parâmetros metabólicos relativos a obesidade estão expressos na tabela 1 .

Em um estudo (3) em crossover, 20 garotos $(15,7 \pm 1,4$ anos $)$ obesos $(106,6 \pm 22 \mathrm{~kg})$ foram submetidos a 3 tipos de tratamentos (baixo, médio e alto IG). No dia anterior ao teste, os participantes ingeri- 
Tabela 1. Descrição de alguns estudos em que foi avaliado o efeito do IG na composição corporal e em parâmetros bioquímicos.

\begin{tabular}{|c|c|c|c|c|c|c|c|c|}
\hline Investigadores & $\mathbf{n}$ & Sexo & $\begin{array}{l}\text { Idade } \\
\text { (anos) }\end{array}$ & Biotipo & Dieta & $\begin{array}{l}\text { Duração } \\
\text { do estudo }\end{array}$ & Efeito & Comentários \\
\hline $\begin{array}{l}\text { Ludwig e cols. } \\
\text { (1999) (2) }\end{array}$ & 20 & M & $15,7 \pm 1,4$ & Obesos & $\begin{array}{l}\text { BIG, MIG e } \\
\quad \text { AIG }\end{array}$ & $\begin{array}{l}\text { Única carga } \\
\text { (24 horas) }\end{array}$ & $\begin{array}{c}\mathrm{BIG}=\downarrow \text { área abaixo da } \\
\text { curva, glicose plasmática, } \\
\text { [ ] insulina, taxa de fome, } \\
\uparrow[\text { ] Glucagon }\end{array}$ & $\begin{array}{c}\text { Dieta BIG apresentou } \downarrow \text { teor } \mathrm{CHO} \text {; } \\
\uparrow \text { PRT e GOR }\end{array}$ \\
\hline $\begin{array}{l}\text { Spieth e cols. } \\
(2000)(11)\end{array}$ & 107 & - & 10 & Obesos & BIG e DRG & $60-$ & $\begin{array}{l}\mathrm{BIG}=\text { maior redução do } \\
\qquad \mathrm{IMC}\end{array}$ & $\begin{array}{c}\text { Dietas diferiram na proporção de } \\
\text { macronutrientes. } \\
\text { Inexistência de avaliação da com- } \\
\text { posição corporal para verificar se a } \\
\text { diminuição do IMC foi devido a } \\
\text { diminuição da gordura corporal. } \\
\text { Não houve monitoramento das } \\
\text { atividades físicas }\end{array}$ \\
\hline
\end{tabular}

\begin{tabular}{|c|c|c|c|c|c|c|c|c|}
\hline $\begin{array}{l}\text { Pawlak e cols. } \\
\text { (2001) (5) }\end{array}$ & 30 & $\begin{array}{l}\text { Ratos } \\
\text { Wistar }\end{array}$ & $\begin{array}{c}6-7 \\
\text { semanas }\end{array}$ & - & $\begin{array}{l}\text { BIG, AIG e } \\
\text { DRG }\end{array}$ & 7 semanas & $\begin{array}{c}\text { Mesmo peso corporal } \\
\text { após os tratamentos, BIG= } \\
\downarrow \text { gordura epididimal; [ ] } \\
\text { Leptina }\end{array}$ & $\begin{array}{l}\text { Limitada extrapolação dos dados } \\
\text { para seres humanos }\end{array}$ \\
\hline $\begin{array}{l}\text { Bouché e cols. } \\
(2002)(6)\end{array}$ & 11 & M & $46 \pm 3$ & $\begin{array}{l}\text { Com } \\
\text { sobrepe- } \\
\text { so }\end{array}$ & $\mathrm{BIG}$ e AIG & 5 semanas & $\begin{array}{c}\text { Mesmo peso corporal } \\
\text { após BIG e AIG; BIG= } \downarrow \\
\text { gordura corporal; } \uparrow \text { massa } \\
\text { magra; } \downarrow \text { mRNA ob, LpL, } \\
\text { HSL }\end{array}$ & $\mathrm{BIG}=\uparrow$ teor de fibras \\
\hline $\begin{array}{l}\text { Ebbeling e cols. } \\
\text { (2003) (17) }\end{array}$ & 16 & $\begin{array}{l}M=5 \\
F=11\end{array}$ & $13-21$ & Obesos & BIG e DRG & 12 meses & $\begin{array}{c}\mathrm{BIG}=\downarrow \mathrm{IMC}, \text { gordura cor- } \\
\text { poral }\end{array}$ & $\begin{array}{c}\text { Dieta BIG apresentou } \downarrow \text { teor } \mathrm{CHO} ; \\
\uparrow \mathrm{PRT} \text { e GOR }\end{array}$ \\
\hline $\begin{array}{l}\text { Ball e cols. } \\
\text { (2003) (1) }\end{array}$ & 16 & $\begin{array}{c}M=8 \\
F=8\end{array}$ & $12-18$ & $\begin{array}{l}\text { Com } \\
\text { sobrepe- } \\
\text { so }\end{array}$ & $\begin{array}{l}\text { TRBIG e } \\
\text { SBIG }\end{array}$ & $\begin{array}{l}3 \text { admissões } \\
\text { de } 24 \mathrm{~h}\end{array}$ & $\begin{array}{l}\text { AIG }=\uparrow \text { área abaixo das } \\
\text { curvas glicêmica e } \\
\text { insulinêmica. } \\
\text { Sem diferença na sen- } \\
\text { sação de fome e na } \\
\text { ingestão }\end{array}$ & $\begin{array}{l}\text { Diferenças na estrutura física das } \\
\text { refeições. } \\
\text { Tempo limitado para a ingestão }\end{array}$ \\
\hline $\begin{array}{l}\text { Sloth e cols. } \\
\text { (2004) (9) }\end{array}$ & 45 & M & $28,9 \pm 1,3$ & $\begin{array}{l}\text { Com } \\
\text { sobre- } \\
\text { peso }\end{array}$ & $\mathrm{BIG}$ e AIG & 10 semanas & $\begin{array}{l}\text { Ingestão alimentar similar } \\
\text { em ambos os grupos. } \\
\text { Mesmo peso e gordura } \\
\text { corporal, massa livre de } \\
\text { gordura e ICQ após os } \\
\text { tratamentos }\end{array}$ & $\begin{array}{l}\text { Uso de registro alimentar. Ambas } \\
\text { as dietas tinham elevado IG (Clas- } \\
\text { sificação de Brand Miller e cols., } \\
\text { 2003). Condições de vida livre; } \\
\text { estudo relativamente longo }\end{array}$ \\
\hline $\begin{array}{l}\text { Alfenas e cols. } \\
(2005)(10)\end{array}$ & 39 & $\begin{array}{c}M=20 \\
F=19\end{array}$ & $24,9 \pm 0,8$ & $\begin{array}{l}\text { Eutrófi- } \\
\cos \end{array}$ & $\mathrm{BIG}$ e AIG & $\begin{array}{l}2 \text { sessões } \\
\text { de } 8 \text { dias }\end{array}$ & $\begin{array}{l}\text { Não houve diferença na } \\
\text { ingestão, percepção de } \\
\text { fome, plenitude gástrica e } \\
\text { desejo de se alimentar }\end{array}$ & $\begin{array}{l}\text { Condições de vida livre; similar } \\
\text { distribuição de macronutrientes; } \\
\text { alimentos consumidos misturados }\end{array}$ \\
\hline
\end{tabular}

M: masculino, F: feminino, IG: índice glicêmico, BIG: baixo índice glicêmico, MIG: Médio índice glicêmico, AIG: Alto índice glicêmico, [ ]: Concentração, CHO: carboidrato, PRT: proteína, GOR: gordura, LpL: Lipase lipoprotéica, HSL: Hormone sensible lipase, mRNA ob: RNA mensageiro do gene ob, $\downarrow$ : menor, $\uparrow$ : maior, DRG: dieta reduzida em gordura, IMC: índice de massa corporal, ICQ: índice cintura-quadril, TRBIG: refeição típica de baixo IG, SBIG: refeição substituta de baixo IG.

ram jantar e ceia de baixo IG. No dia seguinte, o desjejum, contendo alimentos apresentando IG de acordo o tipo de tratamento pré-definido, foi consumidos em 20 minutos. Amostras sanguíneas foram coletadas e a avaliação subjetiva do apetite foi realizada a cada 30 minutos, durante as $\mathbf{5}$ horas, após a ingestão do desjejum. $\mathrm{O}$ almoço, que também foi ingerido em $20 \mathrm{mi}-$ nutos, apresentou o mesmo IG do desjejum. Nas 5 horas seguintes, os participantes puderam ingerir alimentos ad libitum, caso sentissem fome.
Naquele estudo (3), a ingestão de alimentos de baixo IG resultou em menor área abaixo da curva de resposta glicêmica, menor concentração insulínica e níveis de glucagon plasmático maiores, sendo este suprimido após a ingestão das dietas de alto e médio IG. A ingestão da dieta de alto IG resultou em maiores escores de fome e em relação à dieta de baixo IG. Além disso, os participantes sentiram fome mais cedo após o almoço contendo alimentos de alto IG do que após a ingestão da refeição contendo alimentos de baixo IG (3). 
Segundo os autores desse último estudo (3), a hiperinsulinemia e a hipoglucagonemia observadas após a ingestão de alimentos de alto IG tendem a acelerar o metabolismo muscular e hepático, reduzindo a produção de glicose hepática e suprimindo a lipólise. Desta forma, há diminuição dos níveis de glicose e ácidos graxos na circulação. Há, então, aumento dos níveis dos hormônios contra-regulatórios, provocando aumento da fome e da ingestão numa tentativa de restaurar a homeostase energética. Assim, a ingestão crônica de alimentos de alto IG tende a reduzir a oxidação dos nutrientes, fazendo com que os mesmos sejam estocados (12).

No entanto, o estudo citado anteriormente (3) apresenta problemas metodológicos que comprometem a interpretação dos seus resultados. A resposta glicêmica observada após a ingestão dos alimentos é afetada tanto pela qualidade quanto pela quantidade de carboidrato ingerido em uma refeição (13). A dieta de alto IG apresentou maior teor de carboidratos do que a de baixo IG. Assim, a maior área abaixo da curva de resposta glicêmica observada após a ingestão dos alimentos de alto IG em relação aos de baixo IG não pode ser atribuída simplesmente ao efeito do IG. Além disso, dentre os macronutrientes, as proteínas representam o grupo que resulta em maior poder de saciedade (14). Como a dieta de baixo IG apresentou teor de proteínas quase 2 vezes maior do que a dieta de alto IG, a obtenção de menores escores de fome associados à ingestão desse tipo de dieta também já era prevista.

Além desses fatores, deve-se observar que o tempo para a ingestão dos alimentos foi estipulado em 20 minutos. Em condições de vida livre, o tempo estipulado para ingerir as refeições não é necessariamente controlado. Finalmente, Ludwig e cols. (3) sugerem que o tratamento da obesidade baseado na ingestão de alimentos de baixo IG seja norteado pelo consumo abundante de legumes, hortaliças e frutas (alimentos ricos em fibras e de baixo valor calórico), reduzindo a ingestão de alimentos de alto IG (alimentos refinados e que apresentam calorias vazias, como os ricos em açúcar) e moderando a quantidade de lipídeos ingeridos. No entanto, tais orientações retratam as recomendações da FAO/WHO (2003) (15) para que se tenha uma alimentação saudável. A ingestão de alimentos ricos em fibras e de baixa densidade energética tem sido uma das estratégias recomendadas para controle do apetite $(16,17)$. Assim, os benefícios que os autores atribuíram ao IG provavelmente ocorreram em função da ingestão de alimentos com tais características e não, propriamente, pela ingestão de alimentos de baixo IG.
Bouché e cols. (8) realizaram um estudo cujo objetivo foi verificar o efeito da ingestão de alimentos diferindo em IG, durante 5 semanas, no metabolismo de glicose e lipídeos, bem como a massa de gordura corporal, de 11 indivíduos não-diabéticos. Nesse estudo delineado em crossover, refeições de alto ou baixo IG foram ingeridas no desjejum e no almoço. Ao final da 5 semanas, não foram observadas diferenças significantes no total de energia ou na composição de macronutrientes das dietas ingeridas entre os tratamentos. Apesar de não ter sido observada diferença no peso corporal ao final do tratamento, observou-se que a ingestão da dieta de baixo IG resultou na redução da gordura do tronco e aumento da massa livre de gordura. Houve também uma diminuição estatisticamente significante do mRNA ob após o consumo da dieta de baixo IG. A concentração de lipase lipoprotéica no tecido subcutâneo abdominal diminuiu 38\% em baixo IG, mas aumentou $48 \%$ em alto IG. Por meio da enzima HSL (hormone sensible lipase) pode-se avaliar o nível de lipólise celular. No estudo, a ingestão da dieta de baixo IG diminuiu a expressão gênica da HSL no tecido adiposo abdominal. Assim, a diminuição de gordura corporal não se deu por aumento da lipólise mediada pela HSL.

Este estudo apresenta um avanço a respeito do tempo de duração do experimento. Foi o primeiro que buscou avaliar os efeitos do IG por um período maior de tempo. Até então, os estudos tinham avaliado o papel do IG após uma única carga de carboidrato. Porém, os resultados apresentados possuem uma validade questionável, pois não se pode afirmar se estes foram obtidos devido ao efeito do IG dos alimentos ou devido à diferença na quantidade de fibras das dietas testadas. A dieta de baixo IG apresentou teor de fibras $38.7 \%$ maior do que a dieta de alto IG. Assim, a maior perda de gordura corporal neste grupo pode ter ocorrido devido à capacidade das fibras em diminuir a absorção de gordura dietética pelo organismo (18).

Pawlak e cols. (7) realizaram um estudo para comparar o efeito da ingestão de dietas isocalóricas de alto ou de baixo IG ( $45 \%$ carboidrato, $35 \%$ gordura, $20 \%$ proteína) ou de dieta rica em gordura (DRG) (20\% carboidrato, $59 \%$ gordura, $21 \%$ proteína), durante 7 semanas, na deposição de gordura epididimal e nas concentrações de leptina de repouso de ratos Wistar. Os resultados demonstraram que não houve diferença no peso corporal entre os grupos. A massa de gordura epididimal do grupo de baixo IG foi $22 \%$ menor que a do grupo de alto IG e $41 \%$ menor que a do grupo DRG. A leptina plasmática foi maior após o tratamento com alto IG $(11,0 \pm 2,1 \mu \mathrm{g} / \mathrm{l})$ quando 
comparado ao baixo IG $(7,5 \pm 1,0 \mu \mathrm{g} / \mathrm{l})$, sendo esta altamente correlacionada à gordura epididimal. Apesar de sua validade científica, estudos com animais de laboratório apresentam valor limitado no que diz respeito à extrapolação desses dados para seres humanos, em função das diferenças existentes no metabolismo entre tais espécies.

Ebbeling e cols. (19) conduziram um estudo para avaliar o efeito da ingestão de uma dieta de baixa carga glicêmica ou reduzida em gordura no controle da obesidade. Foram prescritas dietas a 16 obesos (13 a 21 anos), os quais foram acompanhados por 12 meses. Durante o estudo, a ingestão calórica dos participantes não foi restrita. Os resultados mostraram que o grupo que ingeriu dieta de baixo IG apresentou maior redução do índice de massa corporal (IMC) e da massa gordurosa. A maior perda da adiposidade observada no grupo de baixo IG foi associada a uma menor sensação de fome e/ou maior saciedade, promovendo uma menor ingestão e um menor peso corporal.

No entanto, as dietas testadas neste estudo apresentaram diferenças na distribuição de macronutrientes. A dieta de baixo IG apresentou menor teor de carboidratos, favorecendo a ocorrência de menores respostas glicêmicas e insulinêmicas. Por outro lado, a dieta reduzida em gordura era na verdade normolipídica (25 a 30\%). O conteúdo de fibras da dieta de baixo IG e a maior palatabilidade da dieta de alto IG também podem ter confundido a interpretação dos resultados obtidos.

Sloth e cols. (10) realizaram um estudo para verificar os efeitos a longo prazo da ingestão ad libi tum de dieta apresentando teor reduzido em gordura e rica em carboidrato de baixo IG $(78,6)$ ou em alto IG $(102,8)$ na ingestão energética, peso e composição corporal de indivíduos com sobrepeso. $\mathrm{O}$ desenho experimental desse estudo de 10 semanas foi randomizado em paralelo. As dietas testadas apresentavam composição de macronutrientes e densidade energética similar. Os indivíduos se apresentaram ao laboratório uma vez por semana para adquirir os alimentostestes, recebiam orientação nutricional individual e registraram todos os alimentos consumidos durante $\mathrm{o}$ estudo.

Os resultados desse estudo (10) indicaram que a ingestão alimentar foi similar em ambos os grupos, não suportando a hipótese de que a ingestão de alimentos de baixo IG proporcionam maior saciedade. Ao final do estudo, também não foram observadas diferenças em relação à perda de peso, gordura corporal, massa livre de gordura e índice de circunferência cintura-quadril entre os grupos.
O estudo citado anteriormente (10) apresentou vantagens em relação a vários outros, por ter sido foi conduzido em condições de vida livre, as dietas ingeridas terem apresentado composição de macronutrientes similar e por ter sido um estudo de longa duração ( 10 semanas). No entanto, uma das limitações desse estudo se deve ao fato de os dados referentes aos alimentos ingeridos durante o estudo terem sido originados a partir do registro alimentar, os quais nem sempre refletem a ingestão real (20). Além disso, todos os alimentos que compunham ambas as dietas testadas apresentaram valores de IG elevados, de acordo com os limites de classificação definidos por Brand Miller e cols. (21).

Spieth e cols. (12) avaliaram o efeito da ingestão de dieta de baixo IG em relação à dieta reduzida em gordura (DRG) no tratamento da obesidade infantil. $\mathrm{O}$ estudo envolveu 107 crianças com idade média de 10 anos. Os participantes receberam aconselhamento dietético e recomendação para aumentarem a prática de atividades físicas. A DRG era hipoenergética (restrição de $250-500 \mathrm{Kcal} \mathrm{em} \mathrm{com-}$ paração à ingestão habitual), sendo limitado o consumo de gorduras, açúcares e de alimentos de alta densidade energética. Tal dieta era composta por $55-60 \%$ de carboidratos, $15-20 \%$ de proteínas, $25-30 \%$ de gorduras. A dieta de baixo IG era baseada no modelo de "Pirâmide Modificada", cuja base é composta por vegetais e frutas, seguidos de proteína, grãos, e cujo ápice é composto por açúcares e alimentos refinados. Essa dieta apresentava $45-50 \%$ de carboidratos, $20-25 \%$ de proteínas e $30-35 \%$ de gorduras. Os participantes foram instruídos a alimentarem-se até se sentirem saciados e a lanchar sempre que sentissem fome. Os resultados mostraram maior redução nos valores de IMC no grupo de baixo IG.

Apesar de a redução do IMC ser um dado interessante, deve-se ressaltar que o ideal seria que mudanças na composição corporal das crianças também tivessem sido avaliadas naquele estudo (12), uma vez que a redução no IMC não reflete necessariamente uma redução no teor de gordura corporal. Naquele estudo, as crianças nem sempre eram orientadas na presença dos pais. Este fato pode ter, de certa forma, comprometido o seguimento das orientações dietéticas e as referentes à realização de atividades físicas pelas crianças. Além disso, a realização de atividades físicas não foi monitorada durante o estudo e as dietas diferiram no padrão de distribuição de macronutrientes.

Ball e cols. (1) realizaram um estudo em crossover para investigar os efeitos metabólicos, hormonais e o efeito na saciedade em 16 adolescentes ( 8 
mulheres e 8 homens, 12-18 anos), com sobrepeso (IMC $\geq$ percentil 95 para a idade e sexo), associado à ingestão de refeição típica de baixo IG (TRBIG), ou refeição substituta de baixo IG (SBIG), ou refeição de alto IG (AIG). Os participantes ingeriram dietas de BIG $(45 \%$ de carboidrato, $25 \%$ de proteína, $30 \%$ de gordura) no jantar e na ceia. No dia seguinte, $o$ desjejum foi ingerido em 15 minutos. Amostras sanguíneas foram coletadas e a avaliação subjetiva da fome foi realizada. $\mathrm{O}$ almoço servido apresentou $\mathrm{O}$ mesmo valor de IG do desjejum. Após o almoço, os participantes realizaram atividades leves e foram instruídos a consumirem lanches que possuíam os mesmos alimentos tanto para o grupo de alto quanto o de baixo IG, os quais eram oferecidos até que os participantes ficassem satisfeitos. A fome foi novamente avaliada subjetivamente. Além disso, avaliouse o período de tempo que os participantes levaram para solicitar o lanche e ingestão voluntária de energia. Os resultados demonstraram maiores valores das áreas abaixo das curvas glicêmica e insulinêmica no grupo AIG. No entanto, não houve diferença na sensação de fome entre as refeições. O lanche foi requisitado mais cedo pelo grupo AIG. Porém, uma vez requerido, não houve diferença na ingestão energética entre os grupos.

No entanto, no estudo anterior, a dieta oferecida ao grupo TRBIG apresentava constituição sólida, enquanto a SBIG era líquida. Diferenças na estrutura física das refeições exercem efeitos sobre o IG e, por isso, dificultam a comparação das respostas metabólicas associadas à ingestão das diferentes refeições (22). O tempo limitado ( 15 minutos) para a ingestão do alimento é outra variável que pode ter afetado a validade dos resultados.

Alfenas e Mattes (11) avaliaram o efeito da ingestão ad libitum de dietas diferindo em IG no apetite e balanço energético. As dietas testadas eram compostas por alimentos apresentando composição de macronutrientes, densidade calórica, teor de fibras e palatabilidade semelhantes. Participaram do estudo 39 indivíduos saudáveis $(24,9 \pm 0,8$ anos, IMC 22,9 $\mathrm{kg} / \mathrm{m}^{2}$ e percentual de gordura $23,5 \%$ ), que consumiram dietas de alto ou de baixo IG ad libtum. A pesquisa foi conduzida em 2 períodos de 8 dias, separados por 15 dias, no mínimo. $\mathrm{O}$ apetite, a glicemia e a insulinemia foram determinadas antes e durante 2 horas após a ingestão do desjejum e almoço, nos dias l e 8. A ingestão energética foi avaliada durante todo o estudo. Os resultados demonstraram que não houveram diferenças na resposta glicêmica e insulinêmica, nos parâmetros subjetivos de fome e na ingestão ener- gética entre os tratamentos. Esse estudo não suporta a hipótese de que a ingestão de alimentos diferindo em IG afeta o apetite de modo distinto.

Este estudo é de grande relevância científica em função da preocupação dos autores em isolar o efeito do IG na ingestão, controlando os possíveis fatores interferentes (teor de macronutrientes, de fibras, densidade energética e palatabilidade dos alimentos testados) nas respostas avaliadas. No referido estudo, as condições de vida livre foram reproduzidas em condições laboratoriais. Os alimentos testados foram ingeridos sem que o tempo designado para a realização das refeições fosse controlado. Além disso, o estudo foi mais longo do que outros estudos dessa natureza $(1,3)$.

\section{CONCLUSÃO}

Os dados aqui apresentados colocam em questionamento a aplicabilidade do IG na prática clínica como uma ferramenta útil para controle da obesidade. Os estudos que atribuem benefícios ao IG dos alimentos sobre o apetite e a composição corporal são experimentos de curta duração, ou realizados com ratos, e na maioria destes a quantidade de macronutrientes e fibras variou entre as dietas testadas. Por outro lado, os estudos que buscaram controlar os fatores dietéticos que comprovadamente exercem um efeito modulador do apetite, retratando ao máximo os eventos que ocorrem em condições de vida livre e que foram conduzidos durante um período de tempo mais longo, não confirmam os efeitos benéficos do IG no controle do apetite, da saciedade e da composição corporal.

\section{REFERÊNCIAS}

1. Ball DS, Keller RK, Moyer-Mileur LJ, Ding YW, Donaldson D, Jackson DW. Prolongation of satiety after low versus moderately high glycemic index meals in obese adolescents. Pediatrics 2003; 111:488-94.

2. Bray GA, Popkin BM. Dietary fat intake does affect obesity! Am J Clin Nutr 1998;68(6):1157-73.

3. Ludwig DS, Majzoub JA, Al-Zahrani A, Dallal GE, Blanco I, Roberts SB. High glycemic index foods, overeating, and obesity. Pediatrics 1999;103(3):E26.

4. Wolever TMS, Jekins DJA, Jekins AL Josse RG. The glycemic index: methodology and clinical implications. Am J Nutr 1991;54:946-54.

5. Pawlak DB, Kushner JA, Ludwig DS. Effects of dietary glycaemic index on adiposity, glucose homoeostasis, and plasma lipids in animals. Lancet 2004;364:778-85.

6. Jenkins DJ, Kendall CW, Augustin LS, Franceschi S, Hamidi $M$, Marchie A, et al. Glycemic index: overview of implications in health and disease. Am J Clin Nutr 2002;76(1):266S-73S.

7. Pawlak DB, Bryson JM, Denyer GS, Brand-Miller JC. High glycemic index starch promotes hypersecretion of insulin and higher body fat in rats without affecting insulin sensitivity. J Nutr 2001;131:99-104. 
8. Bouché $\mathrm{C}$, Rizkalla SW, Jing L, Vidal H, Veronese A, Pacher $\mathrm{N}$ et al. Five-week, low-glycemic index diet decreases total fat mass and improves plasma lipid profile in moderately overweight nondiabetic men. Diabetes Care 2002;25:822-8.

9. Holt SHA, Miller BJ. Increased insulin response to ingested foods are associated with lessened satiety. Appetite 1995;24:43-54.

10. Sloth B, Krog-Mikkelsen I, Flint A, Tetens I, Björck I, Vinoy S, et al. No difference in body weight decrease between a lowglycemicindex and a high-glycemic-index diet but reduced LDL cholesterolafter 10 -wk ad libitum intake of the lowglycemic-index diet. Am J Clin Nutr 2004;80:337-47.

11. Alfenas RCG, Mattes RD. Influence of glycemic index/load on glycemic response, appetite, and food intake in healthy humans. Diabetes Care 2005;28:2123-9.

12. Spieth LE, Harnish JD, Lenders CM, Raezer LB, Pereira MA, Hangen SJ, et al. A low- glicemic index diet in the treatment of pediatric obesity. Arch Pediatr Adolesc Med 2000;154:947-51.

13. Brand-Miller JC, Holt SH, Pawlak DB, McMillan J. Glycemic index and obesity. Am J Clin Nutr 2002;76(1):281S-5S.

14. Jequier E. Pathways to obesity. Int J Obes Relat Metab Disord 2002;26:12S-7S.

15. Word Health Organization. Diet, nutrition and the prevention of chronic disease. Geneva: Word Health Organization, 2003

16. Pai S, Ghugre PS, Udipi SA. Satiety from rice-based, wheatbased and rice-pulse combination preparations. Appetite 2005;44(3):263-71.
17. Rolls BJ, Ello-Martin JA, Tohill BC. What can intervention studies tell us about the relationship between fruit and vegetable consumption and weight management? Nutr Rev 2004;62(1):1-17.

18. Roberts SB, Mc Crory MA, Saltzman E. The influence of dietary composition on energy intake and body weight. J Am Coll Nutr 2002;21:140S-5S.

19. Ebbeling CB, Leidig M, Sinclair KB, Hangen JP, Ludwig DS. A reduced glicemic-load diet in treatment of adolescent obesity. Arch Pediatr Adolesc Med 2003;157:773-9.

20. Flint A, Raben A, Blundell JE, Astrup A. Reproducibility, power and validity of visual analogue scales in assessment of appetite sensations in single test meal studies. Int J Obesity 2000;24:38-48.

21. Brand Miller J, Wolever TMS, Foster-Powell K, Colagiuri S. The New Glycemic Index Revolution: The Authoritative Guide to the Glycemic Index. New York: Marlowe \& Company, 2003.

22. Mattes RD, Rothacker D. Beverage viscosity is inversely related to postprandial hunger in humans. Physiol Behav $2001 ; 74(2): 551-7$.

Endereço para correspondência:

Ana Paula Muniz Guttierres

Av. Antônio Guimarães Peralva, 26

36085-170 Juiz de Fora, MG

E-mail: paulagutti@yahoo.com.br 